\title{
Real-time Estimation of Critical Vehicle Accumulation for Maximum Network Throughput
}

\author{
Konstantinos Ampountolas ${ }^{1}$ and Anastasios Kouvelas ${ }^{2}$
}

\begin{abstract}
Perimeter traffic flow control has recently been found to be a practical and efficient control scheme in mitigating traffic congestion in urban road networks. This control scheme aims at stabilising the accumulation of vehicles of the socalled network fundamental diagram near critical accumulation to achieve maximum network throughput. Nevertheless, the maximum throughput in urban road networks may be observed over a range of accumulation-values. In this work, an adaptive perimeter flow control strategy is proposed that allows the automatic monitoring of the critical accumulation to help maintain the accumulation near the optimal range of accumulationvalues, while network's throughput is maximised. To this end, we design a Kalman filter-based estimation scheme that utilises real-time measurements of circulating flow and accumulation of vehicles to produce estimates of the currently prevailing critical accumulation. We use real data from an urban area with 70 sensors and show that the area exhibits a network fundamental diagram with low scatter. We demonstrate that the fundamental diagram is reproduced under different days but its shape and critical occupancy depend on the applied semi-real-time signal control and the distribution of congestion in the network. Results from the application of the estimation algorithm to the experimental data indicate good estimation accuracy and performance, and rapid tracking behaviour.
\end{abstract}

\section{INTRODUCTION}

Urban road networks without effective means of flow control have been shown to exhibit the throughput-load relationship illustrated in Fig. 1 (e.g. in the form of a flowvehicle accumulation curve). This is the so-called Macroscopic or Network Fundamental Diagram (MFD or NFD) of two-dimensional urban road networks, which provides for network regions under certain regularity conditions (mainly homogeneity in the spatial distribution of congestion and the network topology), a concave, low-scatter relationship between network vehicle accumulations $n$ (veh) and network outflow $q(\mathrm{veh} / \mathrm{h})$ [1], [2], [3], [4]. According to Fig. 1, the typical characteristic of two-dimensional urban road networks is that as the density is increased from zero, the network throughput (circulating flow or trip completion rate) increases to a maximum (flow capacity) and then turns down and decreases sharply to a low value possibly zero (in case of gridlock). Local-area gridlock phenomena can indeed occur but a global gridlock is mostly preventable due to the self-healing (recovery) mechanism of the real transportation networks (as revealed in studies with real data). Note that capacity flow in urban road networks may be observed over a range of vehicle-numbers in contrast to freeway traffic where

\footnotetext{
${ }^{1} \mathrm{~K}$. Ampountolas is with the School of Engineering, University of Glasgow, United Kingdom (E-mail: Konstantinos.Ampountolas@glasgow.ac.uk).

${ }^{2}$ A. Kouvelas is with the Urban Transport Systems Lab, École Polytechnique Fédérale de Lausanne, Switzerland (E-mail: Tasos.Kouvelas@epfl.ch).
}

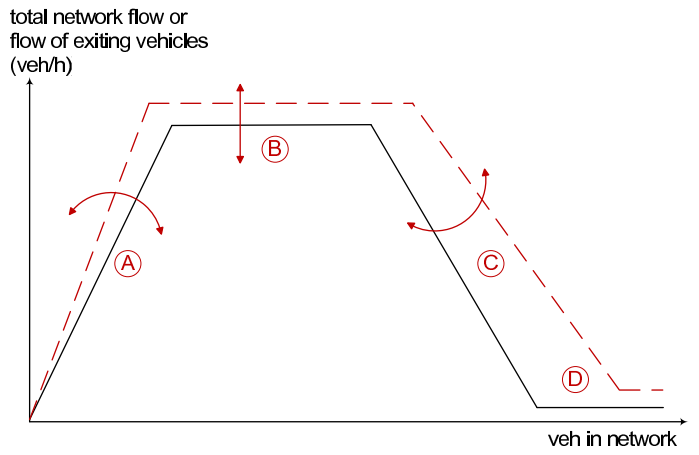

Fig. 1: Theoretical NFD of urban road networks [12].

capacity flow is deemed to occur for a (more or less) specific density value. Physically, when an urban network is congested, some regions may be blocked and significant queue spillback to upstream junctions takes place, which leads to flow degradation and hence infrastructure underutilisation. Hence, flow control mechanisms are necessary to prevent throughput degradation and maintain maximum flow.

The NFD is important for modelling purposes, as details in individual links are not needed to describe the congestion level of cities and its dynamics. It can also be utilised to introduce perimeter flow control policies to improve mobility in homogeneous regions [3], [5], [6], multi-region heterogeneous networks [7], [8], and multi-modal networks [9]. The general idea of a perimeter flow control policy is to "meter" the input flow to the system and to hold vehicles outside the controlled area if necessary, so as to maximise the throughput. Despite these findings for the existence of an NFD, these curves should not be a universal law. Recent works [10], [11] have identified the spatial distribution of congestion in the network affects the scatter of the NFD and its shape. Other works [12] have noticed that flow capacity in urban networks may be observed over a range of accumulation-values (region B in Fig. 1), and thus the critical accumulation $\tilde{n}$ cannot accurately be specified or is subject change due to adaptive signal control [12], [13]. On the other hand, recently developed perimeter flow control [5], [8] relies on pre-specified set values $\hat{n} \approx \tilde{n}$ (see Fig. 2).

These shortcomings of the NFD call for new developments aiming at extending previously proposed feedback perimeter flow control strategies, so as to improve their performance. This paper suggests an adaptive version of the strategies in [5] and [8] that allows for the automatic tracking of the critical accumulation to help maintain the accumulation near the optimal range of accumulation-values, while network's throughput is maximised. We propose a Kalman filter-based 
estimation algorithm that utilises real-time measurements of flow and accumulation of vehicles to produce estimates of the currently prevailing critical accumulation (see [14] for motorway traffic). The paper reports some preliminary results from the evaluation of the estimation algorithm with real experimental data from an urban area with 70 sensors [15].

\section{Methodology}

\section{A. Motivation}

The objective of perimeter flow control or gating is to protect urban regions from over-saturation in the sense of limiting the entrance $q_{\text {in }}$ in the network when it is close to overload. In the case of a homogeneous single-region city the following Proportional-Integral (PI) feedback controller is well suitable for smooth and efficient operations [5]

$$
\begin{aligned}
q_{\text {in }}(k)= & q_{\text {in }}(k-1)-K_{p}[\operatorname{TTS}(k)-\operatorname{TTS}(k-1)] \\
& -K_{I}[\operatorname{TTS}(k)-\widehat{\operatorname{TTS}}]
\end{aligned}
$$

where TTS (veh/h) is the Total Time Spent (a proxy of accumulation); TTS is a desired value of TTS where the Total Traveled Distance (TTD) is maximised (according to the NFD, TTS and TTD are proxies of accumulation and circulating flow, respectively); and, $K_{p}$ and $K_{I}$ are design parameters. This controller aims at stabilising TTS around the selected set point $\widehat{\mathrm{TTS}}$. A well-known feature of the PI regulator is that the regulator error becomes automatically zero, i.e. TTS $=\widehat{\mathrm{TTS}}$ under stationary conditions. For the design of (1) in [5] the corresponding NFD is constructed in terms of TTS and TTD as it has been observed in a field evaluation study in Greece, see Fig. 6 in [16].

In the case of multi-region cities, a single-region policy may induce uneven distribution of vehicles in the regions, and, as a consequence, may invalidate the homogeneity assumption of traffic loads within the urban regions and degrade the total network throughput. In this case, a PI multivariable feedback regulator reads [8]

$$
\boldsymbol{\beta}(k)=\boldsymbol{\beta}(k-1)-\mathbf{K}_{p}[\mathbf{n}(k)-\mathbf{n}(k-1)]-\mathbf{K}_{I}[\mathbf{n}(k)-\hat{\mathbf{n}}]
$$

where $\boldsymbol{\beta}$ is a vector with elements $\beta_{i j}$ the fraction of the gated flow that enters region $j$ from region $i$; $\mathbf{n}$ is a vector with elements $n_{i}$ the accumulation of vehicles in region $i$; $\hat{\mathbf{n}}=(\hat{n})_{i}$ is a set (desired) value for the accumulation of vehicles in each region $i ; \mathbf{K}_{p}$ and $\mathbf{K}_{I}$ are the proportional and integral gains, respectively. This controller aims at stabilising $\mathbf{n}(k)$ around the selected set value $\hat{\mathbf{n}}$. Typically, but not necessarily, $\hat{\mathbf{n}} \approx \tilde{\mathbf{n}}=(\tilde{n})_{i}$ may be selected for each region $i$, in which case the individual regions' output is maximised.

The feedback controllers (1) and (2) aim at stabilising the accumulation of vehicles (or a proxy of accumulation, e.g. TTS, average occupancy or density) of the NFD around a pre-specified set point $\hat{\mathbf{n}} \approx \tilde{\mathbf{n}}$ to achieve maximum network throughput, where $\tilde{\mathbf{n}}$ should be chosen according to Fig. 2 . The design of the feedback controller involves two stages (see [5], [8]): (a) linearisation of the original nonlinear traffic dynamics around $\hat{\mathbf{n}} \approx 90 \% \tilde{\mathbf{n}}$, to allow specification of a nonzero derivative that prevents traffic to visit the congested

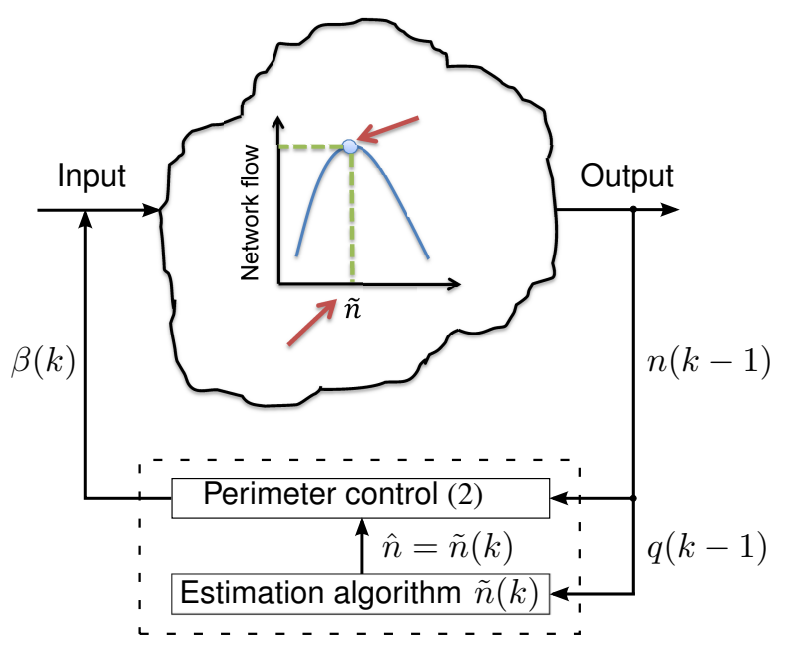

Fig. 2: The adaptive perimeter flow control strategy.

regime; and (b) specification of control gains $\mathbf{K}_{p}$ and $\mathbf{K}_{I}$ (or $K_{p}$ and $K_{I}$ in [5]). Thus the value of $\tilde{\mathbf{n}}$ should be known beforehand and for practical reasons it is estimated visually from the NFD of one representative day. Note that this value is also required for the real-time execution of (2) with given gains $\mathbf{K}_{p}, \mathbf{K}_{I}$ and state measurements $\mathbf{n}(k)$. In this work, we develop an estimation algorithm that allows the automatic monitoring of the critical accumulation and propose an adaptive perimeter control strategy that relies on real-time estimates of critical accumulation to help maintain the accumulation near the optimal range of accumulationvalues, while network's throughput is maximised. The following observations furthermore support the need for an estimation algorithm of critical accumulation and adaptive perimeter traffic flow control:

- The flow capacity in urban road networks may be observed over a range of accumulation-values (see Fig. 1; [12], [5], [8] and the related comments therein).

- The spatial distribution of congestion in the network affectS the scatter of an NFD and its shape [10], [11].

- The critical accumulation $\tilde{n}$ cannot accurately be specified or is subject change due to traffic-responsive signal control (see e.g. [12], [13]), traffic composition (e.g. cars versus buses, see [17]), or non-recurrent day-to-day traffic patterns (as shown later in this paper).

- The location of loop detectors can affect the shape of the diagram and the value of critical accumulation, as the occupancy value is representative in the proximity of the detector and not for the whole link [18], [19].

- In the case of heterogeneous networks with multiple regions of attraction the specification of set points (critical accumulation for each region) would require some care to prevent regions with high density of destinations from entering the congested regime [12].

\section{$B$. An adaptive perimeter flow control strategy}

These shortcomings of the NFD call for the development of an adaptive perimeter flow control strategy that relies on real-time estimates of critical accumulation $\tilde{n}$ (or the critical value of any other parameter of the NFD). To produce realtime estimates of $\tilde{n}(k)$ we utilise measurements of circulating 
flow $q(k-1)$ and accumulation of vehicles $n(k-1)$. Then the estimate $\tilde{n}(k)$ is used as set value in (2) or (1) (we may accordingly estimate occupancy or TTS $)$, i.e. $\hat{n}=\tilde{n}(k)$. The corresponding adaptive control scheme is shown in Fig. 2. Next section presents the estimation algorithm for $\tilde{n}(k)$.

\section{Estimation of Critical Accumulation with Kalman Filter}

The basic idea behind the proposed Kalman filter-based (KF) estimation algorithm is to utilise real-time measurements of circulating flow $q(k-1)$ and accumulation of vehicles $n(k-1)$ to produce estimates of the derivative of the NFD. The derivative of the NFD gives us valuable information about the operation of the network to the stable (positive derivative) or unstable regimes (negative derivative). These estimates may then be used to produce estimates of the currently prevailing critical accumulation $\tilde{n}(k)$, for which the throughput of the network is maximised. Our approach, with some modifications, is similar to [14], [20] for the case of FD of one-dimensional uninterrupted motorway traffic.

To start with, we assume the derivative $\delta(k)$ may be calculated at each time step $k$ by utilising real-time measurements of $q(\kappa)$ and $n(\kappa), \kappa=k-2, k-1$, as follows

$$
\delta(k)=\frac{\Delta q(k-1)}{\Delta n(k-1)+\varepsilon}
$$

where $\Delta q(k-1)=q(k-1)-q(k-2)$ and $\Delta n(k-1)=$ $n(k-1)-n(k-2)$; and $\varepsilon>0$ is a small parameter in the denominator so that possible high-frequency oscillations of the derivative be suppressed. An accurate KF can be derived by calculating the derivative of the NFD around the critical accumulation $\tilde{n}$ where approximately flow capacity is observed [14]. In this case, $\delta(k)$ may be written as

$$
\delta(k)=\frac{q(k)-Q}{n(k)-\tilde{n}}
$$

where $Q$ is the flow around the current estimate $\tilde{n}$. Note that, if $\delta(k) \approx 0$ then flow $q=Q$ may be viewed as the capacity of the NFD. Considering now a filter with two state variables $\mathbf{x}=\left[\begin{array}{ll}\delta & Q\end{array}\right]^{\tau}$ that is described by a random walk

$$
\mathbf{x}(k)=\mathbf{x}(k-1)+\gamma(k)
$$

and one output equation (rearranging in (4))

$$
q^{o}(k)=\boldsymbol{\varphi}(k) \mathbf{x}(k)+\zeta(k)
$$

where $q^{o}(k)$ is the observed output and $\varphi=\left[\left(\begin{array}{ll}n-\tilde{n}) & 1\end{array}\right]\right.$ is the output vector; $\gamma(k)$ and $\zeta(k)$ are independent, zero-mean, Gaussian processes (white-noise) with covariance $\boldsymbol{\Gamma}$ and variance $Z$, respectively. The estimate $\hat{\mathbf{x}}(k)$ that minimises the conditional expectation, given past observation

$$
\boldsymbol{\Pi}(k)=\mathbf{E}\left\{(\hat{\mathbf{x}}(k)-\mathbf{x}(k))(\hat{\mathbf{x}}(k)-\mathbf{x}(k))^{\tau}\right\}
$$

is given by the Kalman filter $(\hat{\mathbf{x}}(0)=\mathbf{E}\{\mathbf{x}(0)\}$ is given)

$$
\hat{\mathbf{x}}(k)=\hat{\mathbf{x}}(k-1)+\mathbf{K}(k-1)\left[q^{o}(k)-\boldsymbol{\varphi}(k) \hat{\mathbf{x}}(k-1)\right]
$$

where $\hat{\mathbf{x}}=\left[\begin{array}{ll}\hat{\delta} & \hat{Q}\end{array}\right]^{\tau}$ and $\mathbf{K}$ is the filter gain. The $\mathrm{KF}$ gain $\mathbf{K}$ depends upon $\boldsymbol{\Gamma}$ and $Z$ and is calculated from [21], [22]

$$
\mathbf{K}_{k-1}=\left[\mathbf{P}_{k-1}+\boldsymbol{\Gamma}\right] \boldsymbol{\varphi}_{k}^{\tau}\left\{\boldsymbol{\varphi}_{k}\left[\mathbf{P}_{k-1}+\boldsymbol{\Gamma}\right] \boldsymbol{\varphi}_{k}^{\tau}+Z\right\}^{-1}
$$

while the error covariance matrix $\mathbf{P}$ is updated according to

$$
\mathbf{P}(k)=\mathbf{P}(k-1)+\boldsymbol{\Gamma}-\mathbf{K}(k-1) \boldsymbol{\varphi}(k)[\mathbf{P}(k-1)+\boldsymbol{\Gamma}]
$$

from the known initial condition $\mathbf{P}(0)=\mathbf{P}_{0}$. This problem of parameter estimation can be seen as a special case of recursive system identification [21]. The recursive filter (8)(10) is executed starting from the given initial conditions.

Our challenge is to devise a scheme for the real-time estimation of the critical accumulation based on the sign (positive or negative) of the estimated derivative $\delta$ from (8) [14]. In the simplest scheme an initial critical occupancy $\tilde{n}(0)$ is chosen; then in each time step $k$ of the algorithm if the corresponding derivative (8) is sufficiently positive (respectively, negative), the new estimate of $\tilde{n}(k)$ is produced by adding (subtracting) an increment $\Delta^{+}$(respectively, $\Delta^{-}$) to the current estimate $\tilde{n}(k-1)$. Nominal values are $\Delta^{+}=$ $1 \%$ and $\Delta^{-}=1.2 \%$ of normalised vehicle accumulation or occupancy. On the other hand, if the value of the derivative $\delta(k)$ is found to be around zero, the new estimate $\tilde{n}(k)$ is set equal to $\tilde{n}(k-1)$. The reason of introducing two increments $\Delta^{+}$and $\Delta^{-}$(in contrast to [14]) is to allow a different rate of increase (respectively, decrease) of the critical accumulation during the onset (respectively, offset) of congestion. In this way, the algorithm allows a fast recovery of the critical accumulation during abnormal traffic conditions in the network (or whenever the diagram is not welldefined). The algorithm could run in the background and should be activated at each control interval $T_{E}$ (a multiple of the time period $T$ of the perimeter control strategy) and only within specific time windows. The algorithmic scheme of the estimation algorithm is illustrated in Algorithm 1.

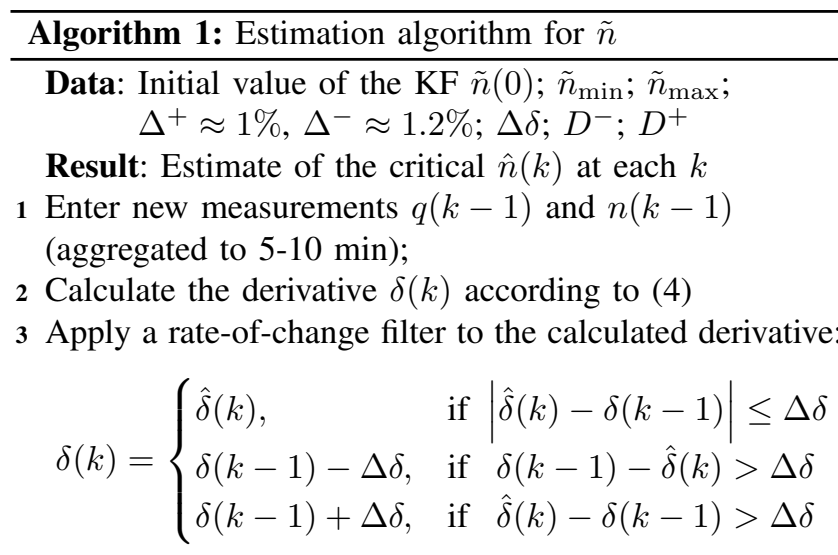

4 Calculate the derivative $\hat{\delta}(k)$ according to (8);

5 Set $\tilde{n}(k)=\Pi\{\tilde{n}(k-1)+s(k)\}$, where $\Pi$ is the projection of the calculated critical occupancy onto $\left[\tilde{n}_{\min }, \tilde{n}_{\max }\right]$ and $s$ is a saturation function as follows

$$
s(k)=\left\{\begin{aligned}
\Delta^{+}, & \text {if } \delta(k)>D^{+} \\
-\Delta^{-}, & \text {if } \delta(k)<D^{-} \\
0, & \text { otherwise }
\end{aligned}\right.
$$

6 Set $\hat{n}(k)=\tilde{n}(k)$ for the perimeter flow control strategy; 7 Set $k:=k+1$; go to step 1 
Step 3 of the algorithm checks if the calculated derivative changes suddenly by a large amount and then returns close to the original value at the next time instant. To this end, a rate-of-change filter is used to limit the maximum allowable change of the filtered derivative to $\Delta \delta>0$. If noise derivatives are not removed by filtering before the calculated derivative is sent to Step 4, the algorithm will produce large, sudden changes in estimated critical accumulation. Step 3 may be omitted or relaxed (via suitable $\Delta \delta$ ) if the available data are aggregated to a low resolution (e.g. 10-20 min).

\section{EXPERIMENTAL DATA ANALYSIS AND RESULTS}

\section{A. Site and data description}

The test site is the central business district (CBD) of Chania (Greece), including about 24 closely spaced signalised junctions and 71 links with lengths varying from 50 to 500 m. Traffic signals are all multiphase operating under the commercial semi-real-time signal control strategy TASS by Siemens. TASS selects, every $15 \mathrm{~min}$, one out of six fixed signal plans (each with different cycle of 60-100 s, splits, and offsets), depending on the traffic conditions in the network, as reflected by the measurements of 17 "strategic" detectors.

The CBD is congested during the weekday's peaks (especially in the summer due to tourism). Traffic conditions in the CBD are quite different, even among weekdays, due to differences in shop opening times. Real flow-occupancy data from 70 loop detectors and spanning one week, were available for the testing of the proposed estimation algorithms. The real data were available from a field evaluation of the TUC/HYBRID signal control strategy and the TASS strategy in May-June 2006 [15]. The data were available in 1.5-min samples of flow and occupancy (\%) and were aggregated to 6-min all-lanes data via averaging of lane occupancies and summing of flows. The next section analyses experimental data collected from the operation of the TASS strategy from June 5 to June 11, 2006. These data are then used in an offline mode to test the proposed estimation scheme.

\section{B. Real data analysis}

We now show with real data that the CBD exhibits an NFD. We demonstrate that the fundamental diagram is reproduced under different traffic conditions (different days) but its shape and critical occupancy depend on the applied signal control and the distribution of congestion in the network.

Fig. 3 depicts experimental data (flow-occupancy time series) for three representative weekdays and a weekend day, and the corresponding NFDs (circulating flow vs. occupancy). Each measurement point on the NFD corresponds to 90 s. Remarkably, Fig. 3 (see NFDs on the right) confirms the existence of an NFD for the CBD with moderate scatter across different days (aggregated data may produce lower scatter, not shown here). It can be seen that flow capacity (around $800 \mathrm{veh} /$ cycle) occur in different occupancies across different days and only a part of region $\mathrm{C}$ is formed (congested regime in Fig. 1). After careful inspection of Fig. 3, the following observations may be highlighted:
- On Monday (see Fig. 3(a)), flow capacity is observed at $\tilde{o} \approx 20 \%$ and a part of the congested regime C (see Fig. 1) of the NFD is actually visited (notice the negative slope).

- On Tuesday and Friday (Figs 3(b)-(c)) where shops are open in the evening (see occupancy 18:00-22:00), flow capacity is observed over a range of occupancies (18-35\% and $20-25 \%$ respectively); this is attributed to the distribution of congestion (cf. flow and occupancy at 18:0022:00) and the applied signal control. The spatiotemporal distribution of congestion may be seen from the average flow from 18:00 to 20:00 on both weekdays where the average occupancy is sub-maximal on Thursday. The impact of applied signal control (TASS) to the NFD on Tuesday and Friday may be inspected from the difference in occupancy levels during the saturated traffic conditions of region B in Fig. 1, i.e. from the highest occupancy that is reached in each day by different signal control plans.

- The NFDs in Figs 3(b)-(c) indicate that TASS maintains the overall throughput to high values (flow capacity) during the heart of the rush on Tuesday (it is seen to reach up to $35 \%$ occupancies in the network, with a fully formed region B, see Fig. 1), but it fails to maintain the same high values of throughput on Friday (although with lower demand) and a part of the congested regime $\mathrm{C}$ of the NFD is visited (notice the negative slope, which is slightly higher than the corresponding slope on Monday). This is attributed to the spatiotemporal distribution of congestion.

- On Saturday (Fig. 3(d)) with low demand a part of the NFD is formed, and flow capacity is deemed at $\tilde{o} \approx 20 \%$.

From the above observations, it is evident that although the CBD exhibits an NFD the critical accumulation cannot accurately be specified or is subject change due to signal control and non-recurrent day-to-day traffic patterns. This calls for real-time estimation of the critical occupancy that exploit the available real-time loop detector measurements in the best possible way, particularly under different traffic conditions. Next section presents some preliminary results on the performance of the proposed estimation algorithm in monitoring the prevailing critical occupancy in the CBD.

\section{Preliminary evaluation of the estimation algorithm}

In this section we present some results on the performance of the proposed estimation algorithm in monitoring the prevailing critical occupancy $\tilde{o}_{c r}$ in the CBD. Although the general algorithmic scheme 1 concerns the real-time estimation of critical accumulation the same algorithm may be used in estimating critical occupancies. For the application of the estimation algorithm to the data in Fig. 3 the following initial values were selected: $o_{\text {act }}(0)>10 \%, \tilde{o}_{\text {cr }}(0)=20 \%$, $\left[\tilde{o}_{\min }, \tilde{o}_{\max }\right]=[10 \%, 30 \%],\left[\Delta^{+}, \Delta^{-}\right]=[1,1.1] \%$. The limits of the derivative were chosen $\left[D^{+}, D^{-}\right]=[100,-60]$ given that only a part of region $\mathrm{C}$ is visited at the heart of rush (notice the negative slope of the NFD in Fig. 3, which is smaller than the corresponding positive slope in region $\mathrm{A}$ ).

Fig. 4 depicts the results for the flow-occupancy data in Fig. 3(b) where the critical occupancy is seen to depend on the applied semi-real-time signal control (the CBD is seen to 

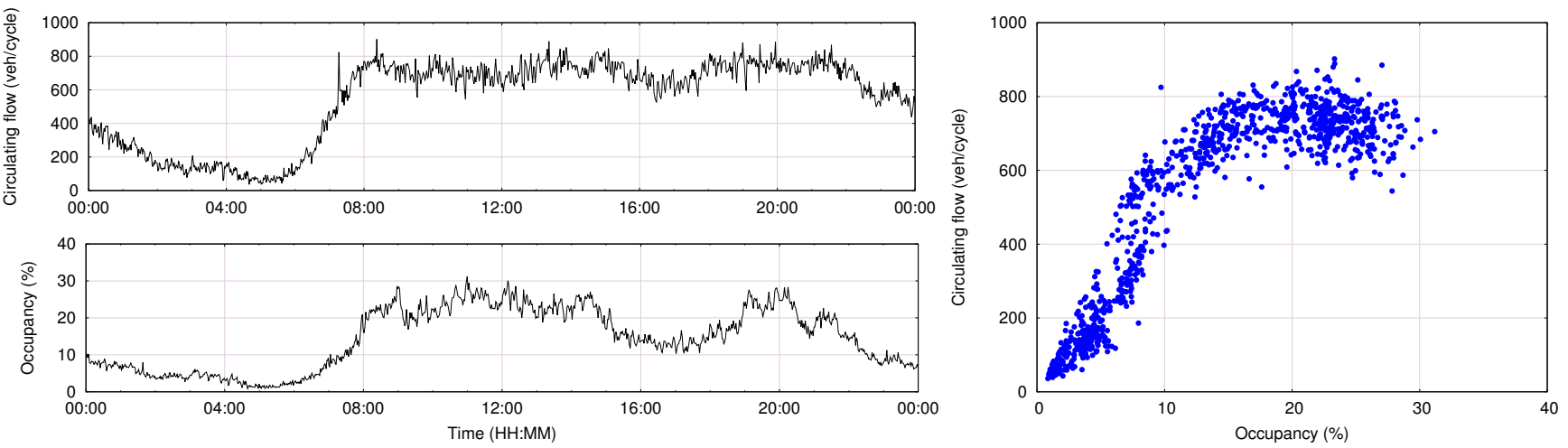

(a) Flow and occupancy time series, and network-wide fundamental diagram on Monday, June 5, 2006.
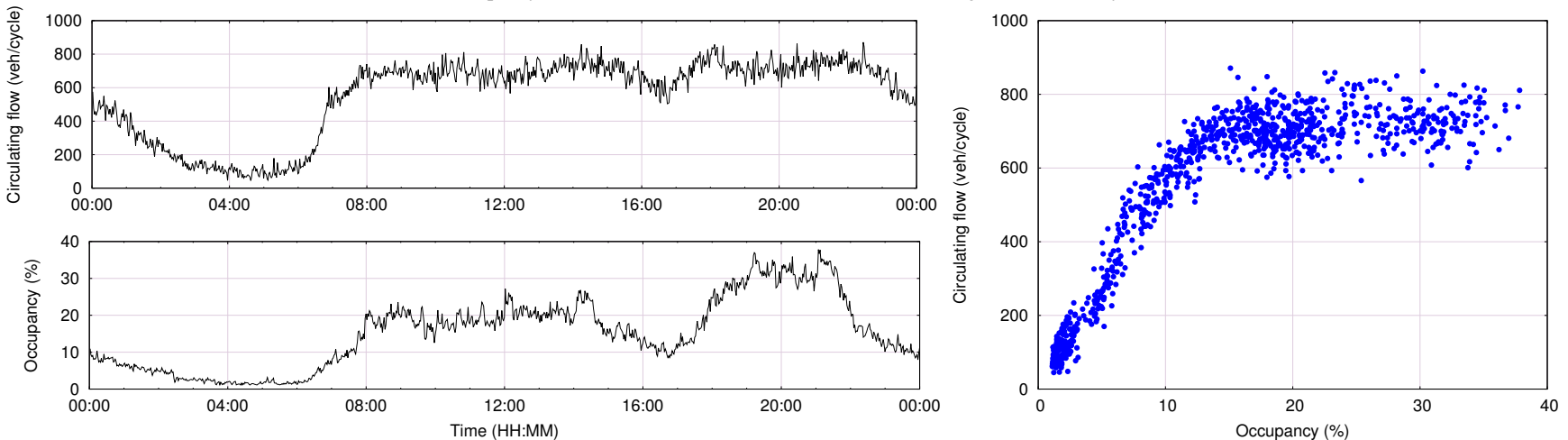

(b) Flow and occupancy time series, and network-wide fundamental diagram on Tuesday, June 6, 2006
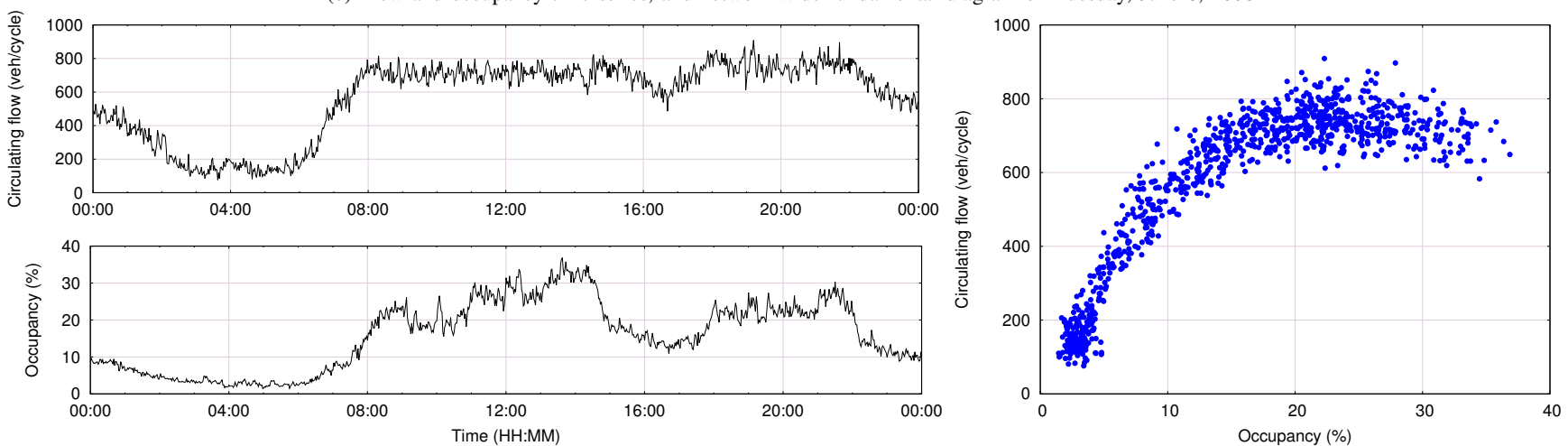

(c) Flow and occupancy time series, and network-wide fundamental diagram on Friday, June 9, 2006
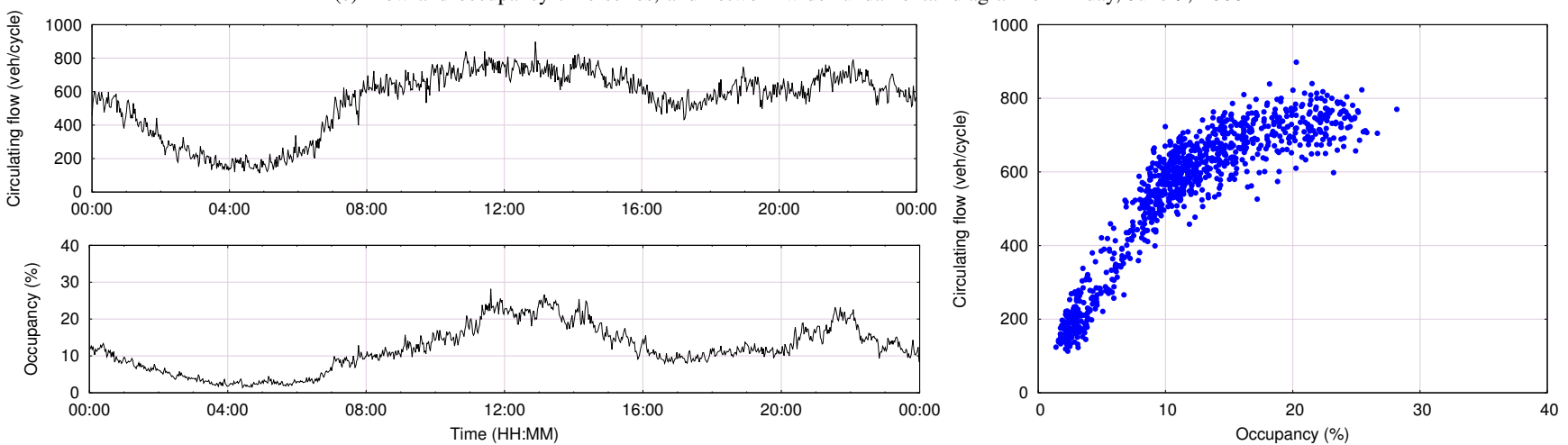

(d) Flow and occupancy time series, and network-wide fundamental diagram on Saturday, June 10, 2006

Fig. 3: Experimental data for three representative weekdays and a weekend day. Each measurement point on the NFD corresponds to $90 \mathrm{~s}$. (a) Regular weekday, flow capacity is observed at $\hat{o} \approx 20 \%$; (b, c) Market is open on Tuesday and Friday evenings (see occupancy 18:00-22:00), with congested traffic conditions and due to adaptive signal control flow capacity is observed over a range of occupancies (18-35\% and 20-25\%, respectively); (d) With low demand a part of the NFD is formed, flow capacity is deemed at $\hat{o} \approx 20 \%$. 

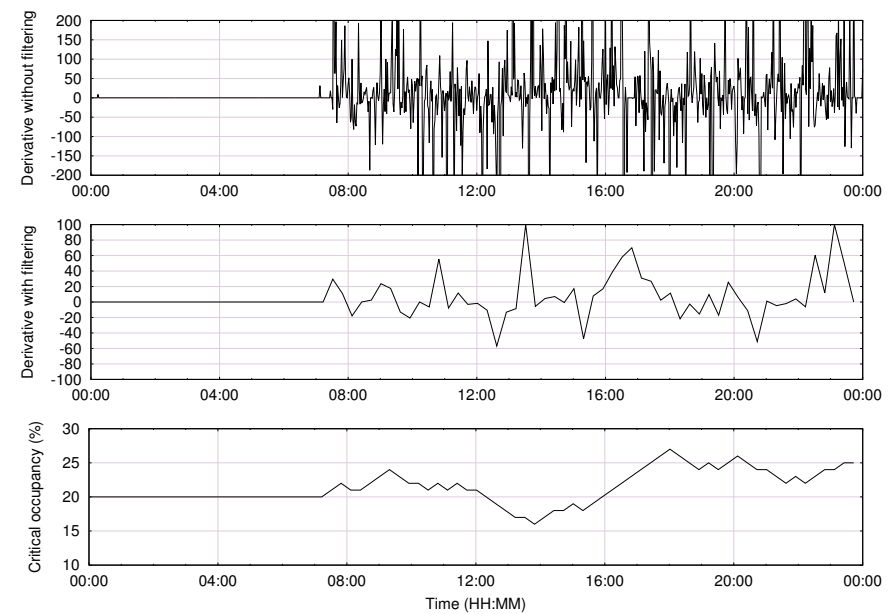

Fig. 4: Derivative estimate (with and without filtering) of the NFD on Tuesday, June 6, 2006; Real-time critical occupancy estimate for flow-occupancy data of Figure 3(b).

reach up to $35 \%$ occupancies, with a fully formed region $\mathrm{B}$ ). The first subfigure displays the obtained derivative (3) before the actual filtering. It can be seen that the derivative tends to oscillate (with high-frequency) between extremes, which is reasonable given that our experimental data are noisy. The second subfigure depicts the estimated derivative after the application of the rate-of-change filter in Step 3 of the algorithm. It is evident that the high-frequency oscillations of the derivative are suppressed after the application of the filter. This allows us to produce very smooth critical occupancy estimates. The third subfigure displays the obtained estimates of critical occupancy. In the morning peak (8:00-12:00) where real occupancy is around 20\% (see occupancy in Fig. 3(b)) the algorithm is seen to estimate a critical occupancy around $21 \%$ (no risk for over saturation). In the evening peak (18:00-22:00) occupancy increases to values around 30\%; as can be seen the algorithm quickly increases $\tilde{o}_{c r}$ during the evening peak and reaches a value of around $25 \%$. Thus the algorithm is seen to estimate quickly and quite accurately the new actual critical occupancy. During the off-peak period the algorithm quickly decreases the critical occupancy to $15 \%$. The goal of the estimation algorithm is to estimate the critical occupancy (given the derivative of the NFD) thus it does not track the actual occupancy. Relocating critical occupancy outside region B (Fig. 1) will jeopardise the performance of the proposed adaptive perimeter flow control strategy ( $\tilde{o}$ is an input for regulator (2), see Fig. 2).

\section{CONCLUSIONS}

In this work, we proposed a Kalman filter-based estimation scheme that utilises real-time measurements of circulating flow and accumulation of vehicles to produce estimates of the currently prevailing critical accumulation. The developed estimation algorithm coupled with the proposed adaptive perimeter flow control strategy may be valuable whenever the NFD is not well defined and the critical accumulation cannot accurately be specified. We used real-data from a CBD with 70 sensors and showed that the urban area exhibits an NFD with low scatter. We demonstrated that the NFD is reproduced under different days but its shape and critical occupancy depend on the applied semi-realtime signal control and the distribution of congestion in the network. Preliminary evaluation results of the estimation algorithm with the experimental data indicated good estimation accuracy and performance.

\section{REFERENCES}

[1] J. W. Godfrey, "The mechanism of a road network," Traffic Eng Control, vol. 11, no. 7, pp. 323-327, 1969.

[2] S. Ardekani and R. Herman, "Urban network-wide traffic variables and their relations," Transport Sci, vol. 21, no. 1, pp. 1-16, 1987.

[3] C. F. Daganzo, "Urban gridlock: Macroscopic modeling and mitigation approaches," Transport Res B-Meth, vol. 41, no. 1, pp. 49-62, 2007.

[4] N. Geroliminis and C. F. Daganzo, "Existence of urban-scale macroscopic fundamental diagrams: Some experimental findings," Transport Res B-Meth, vol. 42, no. 9, pp. 759-770, 2008.

[5] M. Keyvan-Ekbatani, A. Kouvelas, I. Papamichail, and M. Papageorgiou, "Exploiting the fundamental diagram of urban networks for feedback-based gating," Transport Res B-Meth, vol. 46, no. 10, pp. 1393-1403, 2012.

[6] J. Haddad and A. Shraiber, "Robust perimeter control design for an urban region," Transport Res B-meth, vol. 68, pp. 315-332, 2014.

[7] N. Geroliminis, J. Haddad, and M. Ramezani, "Optimal perimeter control for two urban regions with macroscopic fundamental diagrams: A model predictive approach," IEEE Trans Intell Transp Syst, vol. 14, no. 1, pp. 348-359, 2013.

[8] K. Aboudolas and N. Geroliminis, "Perimeter and boundary flow control in multi-reservoir heterogeneous networks," Transport Res BMeth, vol. 55, pp. 265-281, 2013.

[9] K. Ampountolas, N. Zheng, and N. Geroliminis, "Robust control of bimodal multi-region urban networks: An LMI optimisation approach," in Proc. 17th IEEE Int Conf Intell Transp Syst, Qingdao, China, October 8-11, 2014.

[10] A. Mazloumian, N. Geroliminis, and D. Helbing, "The spatial variability of vehicle densities as determinant of urban network capacity," Philos TR R Soc A, vol. 368, no. 1928, pp. 4627-4647, 2010.

[11] N. Geroliminis and J. Sun, "Properties of a well-defined macroscopic fundamental diagram for urban traffic," Transport Res B-Meth, vol. 45, pp. 605-617, 2011.

[12] K. Aboudolas, M. Papageorgiou, A. Kouvelas, and E. Kosmatopoulos, "A rolling-horizon quadratic-programming approach to the signal control problem in large-scale congested urban road networks," Transport Res C-Emer, vol. 18, no. 5, pp. 680-694, 2010.

[13] N. Geroliminis and B. Boyaci, "The effect of variability of urban systems characteristics in the network capacity," Transport Res BMeth, vol. 46, no. 10, pp. 1607-1623, 2012.

[14] E. Smaragdis, M. Papageorgiou, and E. Kosmatopoulos, "A flowmaximizing adaptive local ramp metering strategy," Transport Res BMeth, vol. 38, no. 3, pp. 251-270, 2004.

[15] A. Kouvelas, K. Aboudolas, M. Papageorgiou, and E. Kosmatopoulos, "A hybrid strategy for real-time traffic signal control of urban road networks," IEEE Trans Intell Transp Syst, vol. 12, no. 3, pp. 884-894, 2011.

[16] V. Dinopoulou, C. Diakaki, and M. Papageorgiou, "Application and evaluation of the signal traffic control strategy TUC in Chania," Journal Intell Transport Syst, vol. 9, no. 3, pp. 133-143, 2005.

[17] N. Geroliminis, N. Zheng, and K. Ampountolas, "A three-dimensional macroscopic fundamental diagram for mixed bi-modal urban networks," Transport Res C-Emer, vol. 42, pp. 168-181, 2014.

[18] C. Buisson and C. Ladier, "Exploring the impact of homogeneity of traffic measurements on the existence of macroscopic fundamental diagrams," Transport Res Rec, no. 2124, pp. 127-136, 2009.

[19] L. Leclercq, N. Chiabaut, and B. Trinquier, "Macroscopic fundamental diagrams: A cross-comparison of estimation methods," Transport Res B-Meth, vol. 62, pp. 1-12, 2014.

[20] E. Kosmatopoulos, M. Papageorgiou, D. Manolis, J. Hayden, R. Higginson, K. McCabe, and N. Rayman, "Real-time estimation of critical occupancy for maximum motorway throughput," Transport Res Rec, vol. 1959, pp. 65-76, 2006.

[21] L. Ljung and T. Söderström, Theory and Practice of Recursive Identification. Cambridge, Massachusetts: MIT Press, 1983.

[22] M. Papageorgiou, Optimierung, 2nd ed. Munich, Germany: Oldenbourg, 1996. 